\title{
A NOTE ON REPRESENTING MEASURES
}

\section{N. T. PECK $^{1}$}

Let $K$ be a closed bounded convex subset of a normed linear space $E$, and denote the set of extreme points of $K$ by ext $K$. If ext $K$ is a nonvoid Borel set, we say that a point $x$ of $K$ is representable in $K$ if there is a probability measure $\mu$ on $K$ (regular, nonnegative Borel measure of total mass 1 ) supported on ext $K$ with the following property: for every continuous linear functional $\Psi$ on $E$,

$$
\Psi(x)=\int_{\text {ext } K} \Psi(y) d \mu(y) .
$$

Let $D$ be the closed unit disc in the complex plane and let $C(D)$ be the space of continuous complex-valued functions on $D$, with the usual sup norm. If $U$ is the closed unit ball of $C(D)$, a special case of a result of Phelps [1] is that $U$ is the closed convex hull of ext $U$; in view of this, the following example seems of interest:

TheOREM. Let $f$ in $U$ be defined by $f(z)=z$. Then $f$ is not representable in $U$.

Proof. Let $B=\{z \in D:|z|=1\}$; let $\left\{z_{n}\right\}$ be a sequence dense in $B$. Define linear functionals $\Psi_{n}$ on $C(D)$ by $\Psi_{n}(g)=g\left(z_{n}\right)$. Assume that $\mu$ is a measure which represents $f$; then for each $n$ we have

$$
z_{n}=\Psi_{n}(f)=\int_{\text {ext } U} \Psi_{n}(g) d \mu(g)=\int_{\text {ext } U} g\left(z_{n}\right) d \mu(g) .
$$

Multiplying by $z_{n}^{-1}$, we have

$$
1=\int_{\text {ext } U} z_{n}^{-1} g\left(z_{n}\right) d \mu(g) .
$$

If $\phi_{n}(g)=z_{n}^{-1} g\left(z_{n}\right),\left|\phi_{n}(g)\right| \leqq 1$ for all $g$ in $U$; since $\mu$ is a probability measure, it follows easily from equation $\left(^{*}\right)$ that $U_{n}=\{g \in \operatorname{ext} U$ : $\left.z_{n}^{-1} g\left(z_{n}\right) \neq 1\right\}$ has $\mu$-measure 0 . Hence $\mu\left(\cup_{n=1}^{\infty} U_{n}\right)=0$; it then follows that there is $h$ in ext $U$ such that $h\left(z_{n}\right)=z_{n}$ for all $n$. Since $h$ is continuous and $\left\{z_{n}\right\}$ is dense in $B$, it follows that $h(z)=z$ for all $z$ in $B$; since $h$ is in ext $U,|h(z)|=1$ for all $z$ in $D$. Since $B$ is not a retract of $D$ we have a contradiction and the proof is complete.

Received by the editors September 29, 1965.

1 Research for this note was partially supported by the National Science Foundation under grant NSF-GP-1814. 


\section{BIBLIOGRAPHY}

1. R. R. Phelps, Extreme points in function algebras, Duke Math. J. 32 (1965), 267-277.

YaLe University

\section{ON THE OSCILLATION OF SUMS OF RANDOM VARIABLES}

\section{L. HANSON ${ }^{1}$ AND MELVIN KATZ ${ }^{2}$}

The object of this note is to prove the following theorem which may be interpreted as stating that sums of independent and identically distributed random variables do not oscillate too violently.

THEOREм. Let $\left\{X_{k}: k \geqq 1\right\}$ be a sequence of independent and identically distributed random variables and let $S_{n}=\sum_{k=1}^{n} X_{k}$. The series $\sum_{n=1}^{\infty}(-1)^{n} n^{-1} P\left(S_{n}>0\right)$ is convergent.

Proof. Let $l=P\left\{\bigcap_{\mathbf{k}=1}^{\infty}\left[S_{k}>0\right]\right\}$ and $l^{\prime}=P\left\{\bigcap_{\mathbf{k}=1}^{\infty}\left[S_{k} \leqq 0\right]\right\}$. It is known, [2], that for $|t|<1$

$$
\sum_{n=0}^{\infty} t^{n} P\left\{\bigcap_{k=1}^{n}\left[S_{k}>0\right]\right\}=\exp \left\{\sum_{k=1}^{\infty} t^{k} k^{-1} P\left[S_{k}>0\right]\right\} .
$$

If $l=0$ then $\sum_{n=0}^{\infty}(-1)^{n} P\left\{\bigcap_{k=1}^{n}\left[S_{k}>0\right]\right\}$ is convergent. It follows from (1), and the Littlewood Tauberian Theorem that

$$
\sum_{n=1}^{\infty}(-1)^{n} n^{-1} P\left(S_{n}>0\right)
$$

is convergent. Similarly, if $l^{\prime}=0$ then $\sum_{n=1}^{\infty}(-1)^{n} n^{-1} P\left(S_{n} \leqq 0\right)$ is convergent which implies $\sum_{n=1}^{\infty}(-1)^{n} n^{-1} P\left(S_{n}>0\right)$ is convergent.

For the case $l>0$ and $l^{\prime}>0$ we proceed differently. $\sum_{n=1}^{\infty}(-1)^{n} n^{-1}$ is convergent and thus it suffices to prove

$$
\sum_{n=1}^{\infty}\left|P\left(S_{n}>0\right)-P\left(S_{n+1}>0\right)\right|<\infty .
$$

However this last sum is bounded by $\sum_{n=1}^{\infty}\left\{P\left(S_{n}>0, S_{n+1} \leqq 0\right)\right.$ $\left.+P\left(S_{n} \leqq 0, S_{n+1}>0\right)\right\}$. Since $l>0$ and $l^{\prime}>0$ it follows by a standard

Received by the editors July 22, 1965.

1 Research supported by the Air Force Office of Scientific Research.

2 Supported in part by National Science Foundation Grant GP-1816. 